\title{
On-drug and drug-free remission by baseline symptom duration: abatacept with methotrexate in patients with early rheumatoid arthritis
}

\author{
Vivian P. Bykerk ${ }^{1}\left[\right.$. Gerd R. Burmester ${ }^{2} \cdot$ Bernard G. Combe $^{3} \cdot$ Daniel E. Furst $^{4} \cdot$ Tom W. J. Huizinga $^{5}$. \\ Harris A. Ahmad ${ }^{6} \cdot$ Paul Emery 7,8
}

Received: 15 June 2018 / Accepted: 5 October 2018 / Published online: 20 October 2018

(c) The Author(s) 2018, corrected publication 2018

\begin{abstract}
Clinical outcomes in patients with early rheumatoid arthritis (RA) were assessed by baseline symptom duration in the Assessing Very Early Rheumatoid arthritis Treatment trial (ClinicalTrials.gov; NCT01142726). Patients with early, active RA were randomized to subcutaneous (SC) abatacept $125 \mathrm{mg} /$ week plus methotrexate (MTX), SC abatacept alone, or MTX monotherapy for 12 months. All RA treatments were withdrawn after 12 months in patients with Disease Activity Score in 28 joints (C-reactive protein; DAS28-CRP) $<3.2$. In this post hoc analysis, the proportion of patients achieving protocoldefined remission (DAS28-CRP <2.6) or improvement in physical function at 12 and at both 12 and 18 months was assessed according to symptom duration ( $\leq 3$ months, $>3$ to $\leq 6$ months, or $>6$ months) and treatment group. No clinically significant differences were seen in baseline demographics or characteristics across symptom duration groups. Irrespective of baseline symptom duration, a numerically higher proportion of abatacept plus MTX-treated patients achieved DAS-defined remission at month 12 and sustained remission at month 18 compared with MTX monotherapy. A numerically higher proportion of abatacept plus MTX-treated patients with symptom duration $\leq 3$ months maintained DAS-defined remission after complete treatment withdrawal from 12 to 18 months compared with longer symptom duration groups. This subgroup also had the fastest onset of clinical response (DAS28-CRP <2.6) after initiation of treatment. Health Assessment Questionnaire-Disability Index response was similar regardless of baseline symptom duration. Overall, symptom duration of $\leq 3$ months was associated with a faster onset of clinical response and higher rates of drug-free remission following treatment with abatacept plus MTX.
\end{abstract}

Keywords Rheumatoid arthritis · Biological therapy $\cdot$ Antirheumatic agents $\cdot$ Clinical trial $\cdot$ Abatacept

Electronic supplementary material The online version of this article (https://doi.org/10.1007/s00296-018-4173-3) contains supplementary material, which is available to authorized users.

Vivian P. Bykerk

bykerkv@hss.edu

1 Department of Rheumatology, Hospital for Special Surgery, Weill Cornell Medical College, 535 East 70th St, New York, NY 10021, USA

2 Department of Rheumatology and Clinical Immunology, Charité-University Medicine Berlin, Berlin, Germany

3 Department of Rheumatology, Service d'Immuno-Rheumatologie, Montpellier, France

4 Department of Medicine, University of California Los Angeles, Los Angeles, CA, USA
5 Department of Rheumatology, Leiden University Medical Center, Leiden, Netherlands

6 Headquarters Medical Immunology, Bristol-Myers Squibb, Princeton, NJ, USA

7 Leeds Institute of Rheumatic and Musculoskeletal Medicine, University of Leeds, Leeds, UK

8 NIHR Leeds Musculoskeletal Biomedical Research Unit, Leeds Teaching Hospitals NHS Trust, Leeds, UK 


\section{Introduction}

Rheumatoid arthritis (RA) is a chronic, progressive, inflammatory disease characterized by chronic joint inflammation followed by structural damage [1]. An optimum 'window of opportunity' has been proposed in early RA, during which treatment can alter the disease course before inflammatory processes become more established [2]. In support of this theory, patients with RA of longer disease duration generally do not respond as well to treatment with a biologic disease-modifying antirheumatic drug (bDMARD) as those with a shorter duration of disease [3], with earlier treatment providing better disease control than later treatment [4]. In patients with well-controlled disease, sustained remission after the cessation of immunomodulatory medications could indicate to clinicians that disease modification had been achieved [5].

The Assessing Very Early Rheumatoid arthritis Treatment (AVERT) study examined the ability of subcutaneous (SC) abatacept plus methotrexate (MTX) vs MTX monotherapy to induce clinical remission in patients with early RA after 12 months of treatment, and also assessed whether sustained remission could be achieved 6 months after withdrawal of all RA therapy (month 18) [5]. Compared with patients treated with MTX alone, a greater proportion of patients treated with SC abatacept plus MTX achieved protocol-defined remission Disease Activity Score in 28 joints with $\mathrm{C}$ reactive protein (DAS28-CRP) $<2.6$ at month 12 and also sustained drugfree remission at month 18 [5]. Although not statistically powered for comparison with combination therapy or MTX, a SC abatacept monotherapy treatment group was also included. In both abatacept treatment arms, the proportions of patients with sustained DAS-defined remission following treatment withdrawal were numerically higher in patients who had shorter symptom duration ( $<6$ months), lower baseline DAS28-CRP and lower Health Assessment Questionnaire -Disability Index (HAQ-DI) - an effect not observed in the MTX treatment arm [5].

AVERT was the first trial to investigate the possibility of achieving absolute drug-free remission after withdrawal of all RA therapies. As drug-free remission is an attractive treatment goal in early RA, understanding whether there is an optimum treatment window to initiate therapy has important implications for clinical practice. The AVERT trial provides the opportunity to examine outcomes in patients with early RA of varying duration, when symptom duration is well defined across groups. This longitudinal post hoc analysis was performed to examine the impact of baseline symptom duration on achieving remission and sustained drug-free remission in patients with early RA enrolled in the AVERT trial.

\section{Patients and methods}

\section{Study design and patient population}

We performed post hoc analyses on data from the AVERT study (NCT01142726). Full study design, methods, inclusion criteria and primary results have been described previously [5]. Briefly, AVERT was a phase $3 \mathrm{~b}$, randomized, active-controlled trial consisting of a 12-month treatment period and a 12-month withdrawal period [5]. Patients were randomized 1:1:1 to SC abatacept $125 \mathrm{mg} /$ week plus MTX, SC up to $20 \mathrm{mg} /$ week, abatacept monotherapy or MTX monotherapy for 12 months. At month 12, all RA treatment was withdrawn in patients with DAS28-CRP $<3.2$. Patients enrolled in AVERT had active clinical synovitis of $\geq 2$ joints for $\geq 8$ weeks with persistent symptoms for $\leq 2$ years, DAS28-CRP $\geq 3.2$ and anti-CCP2 antibody positivity. Patients were MTX naïve or had received MTX $(\leq 10 \mathrm{mg}$ ) week) for $\leq 4$ weeks with no MTX for 1 month prior to enrolment.

\section{Outcomes and statistical analysis}

In this post hoc analysis, outcomes were analyzed in patients grouped by symptom duration (defined as the duration of persistent symptoms at baseline) of $\leq 3$ months, $>3$ to $\leq 6$ months, or $>6$ months, as these time points are significant in determining whether a patient is adequately responding to therapy, and also by treatment group [6, 7]. Baseline demographic data and disease characteristics for each subgroup were calculated. The proportion of patients achieving remission (DAS28-CRP, Boolean, Simplified Disease Activity Index [SDAI] and Clinical Disease Activity Index [CDAI]), or improvements in physical function (HAQ-DI response) were calculated at monthly intervals up to month 12 during the treatment period, and then at months 13, 14, 15, and 18 during the withdrawal period. Patients in remission at both months 12 and 18 were considered to have sustained drugfree remission. Odds ratios and $P$ values were generated using a Logistic Regression test which adjusted for treatment group, baseline DAS28-CRP value and corticosteroid use at baseline (yes/no). Definitions of remission for the different disease activity measures were: DAS28-CRP $<2.6$; Boolean: tender joint count $28 \leq 1$, swollen joint count $28 \leq 1$, patient 
global assessment of disease activity $(0-10 \mathrm{~cm}) \leq 1$ and high-sensitivity $\mathrm{CRP} \leq 1 \mathrm{mg} / \mathrm{dl}$; $\mathrm{SDAI} \leq 3.3$ and $\mathrm{CDAI} \leq 2.8$. Improvement in physical function (HAQ-DI response) was defined as a reduction in HAQ-DI score of $\geq 0.3$ units from baseline as this had been a previously defined threshold for analysis in this trial [5].

Missing DAS-defined remission or HAQ-DI response data, not due to premature discontinuation and not at treatment period day 1 or month 18 , was imputed as DAS-defined remission or HAQ-DI response if the missing value was between two observed DAS-defined remissions or HAQDI responses (if not, the patient was considered not in remission).

\section{Results}

\section{Patient disposition}

The AVERT intent-to-treat population comprised 351 patients with severe, active, early RA: 119 were randomized to receive abatacept plus MTX, 116 to abatacept monotherapy and 116 to MTX monotherapy. No clinically significant or consistent differences were seen in baseline demographic data and clinical characteristics when patients were grouped by treatment and symptom duration; however, both the proportion of patients from South America and the mean baseline symptom duration were lowest for patients with $\leq 3$ months' symptom duration, and the proportion of male patients was higher in the $>6$ months' group than in the other groups (Supplementary Table 1).

\section{Proportion of patients in remission and sustained remission}

Irrespective of baseline symptom duration, a higher proportion of patients receiving abatacept plus MTX achieved DAS-defined remission at month 12 and sustained remission at month 18 compared with those receiving MTX monotherapy (Supplementary Table 2). Numerically greater treatment differences in sustained remission (measured at 12-18 months) following withdrawal of all treatment were observed for patients with $\leq 3$ months' symptom duration vs those with longer symptom durations with both abatacept plus MTX and abatacept monotherapy (Fig. 1a,b). Patients treated with abatacept plus MTX who had symptom durations of $\leq 3$ months also had the numerically fastest onset of DAS28-CRP-defined remission (Fig. 1a, Supplementary Table 3 ). Among patients with $\leq 3$ months' symptom duration, as early as day 29 , a numerically higher proportion of patients receiving abatacept plus MTX $(25.0 \%)$ achieved DAS-defined remission compared with patients receiving abatacept alone $(2.8 \%)$ or MTX alone $(8.3 \%)$. The rate of DAS-defined remission at day 29 for patients receiving abatacept plus MTX with $\leq 3$ months' symptom duration $(25.0 \%)$ was greater than for patients receiving this treatment with symptom durations of $>3$ to $\leq 6$ months (11.8\%) and $>6$ months $(6.1 \%)$.

In the abatacept plus MTX group, a numerically higher proportion of patients maintained DAS-defined remission at 18 months during the drug-free period in the subgroup with symptom durations of $\leq 3$ months $(33.3 \%$ [95\% CI: 17.9, 48.7]) compared with those with symptom durations of $>3$ to $\leq 6$ months $(14.7 \%[5.0,31.1])$ and $>6$ months $(10.2 \%$ $[3.4,22.2])$; these differences were not seen in patients receiving abatacept or MTX alone (Fig. 1a-c).

Similar results to those seen for DAS28-CRP-defined remission were observed when using CDAI, SDAI or Boolean measures of disease remission (Supplementary Figs. 1-3).

The proportion of patients with improvements in physical function (HAQ-DI response) was comparable, irrespective of baseline symptom duration, with a numerically greater proportion of patients with an improvement with abatacept plus MTX compared with abatacept monotherapy and MTX monotherapy. This treatment difference was seen as early as day 29 and persisted up to 6 months after treatment withdrawal (Fig. 2a-c).

\section{Discussion}

The results of this post hoc analysis demonstrate that, irrespective of symptom duration at treatment initiation, patients with early RA treated with abatacept plus MTX achieve higher rates of remission and numerically greater proportions of patients with improvements in physical function compared with MTX monotherapy at 12 months. Furthermore, the fastest onset of DAS28-CRP-defined remission and highest rates of drug-free sustained remission were seen in patients receiving abatacept plus MTX who had the shortest symptom duration ( $\leq 3$ months). HAQ-DI response (improvement $\geq 0.3$ ), however, was not affected by baseline symptom duration. These results in subpopulations, based on baseline symptom duration, are consistent with the results seen for the overall AVERT population [5], but demonstrate an additional benefit of very early treatment in terms of achieving remission on drug as well as maintaining 

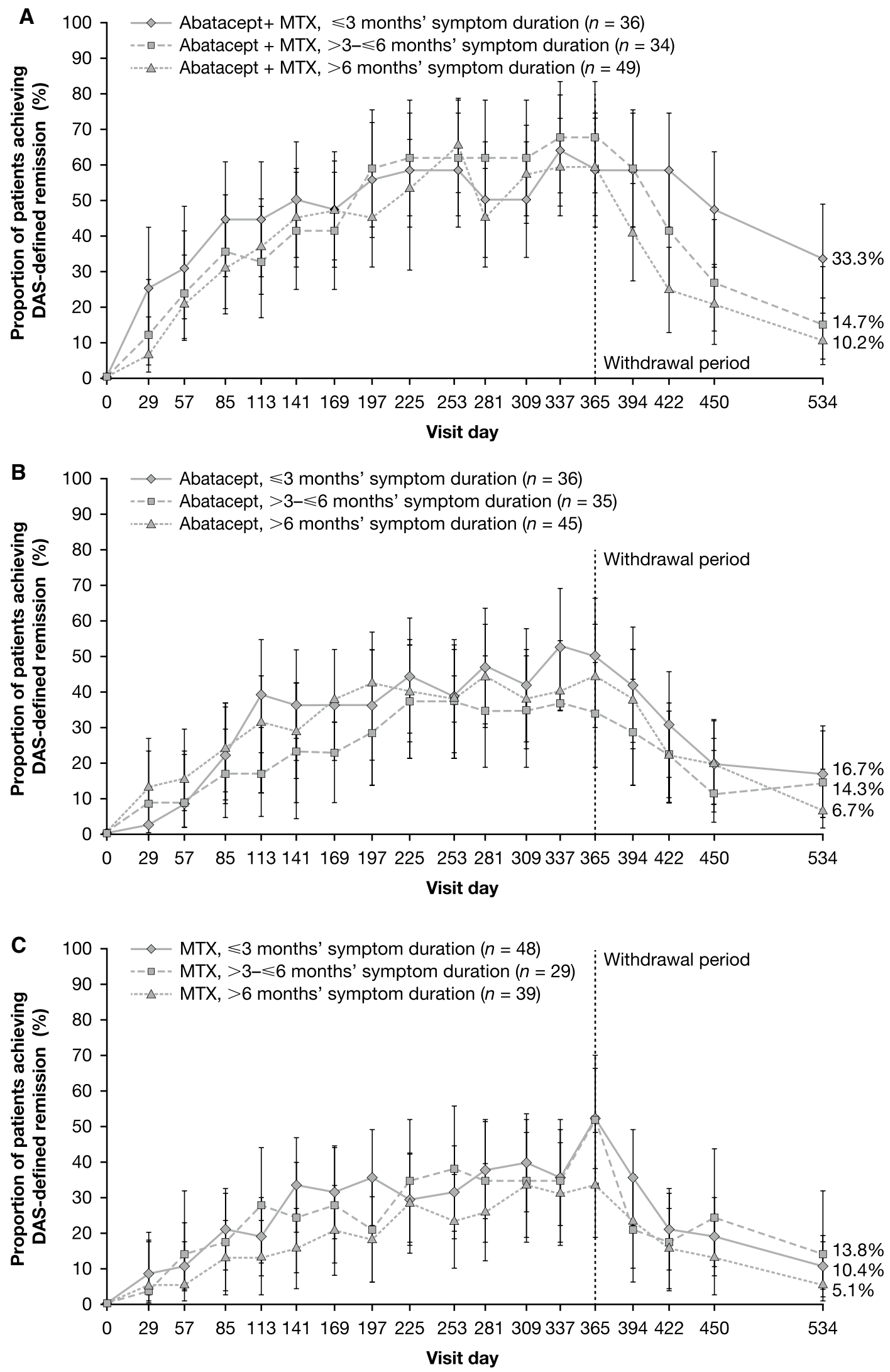
४Fig. 1 Proportion of patients achieving DAS-defined remission over time by symptom duration in each treatment arm: a abatacept + MTX, b abatacept monotherapy, c MTX monotherapy. Error bars represent 95\% CI. DAS-defined remission is defined as DAS28-CRP $<2.6$. DAS28-CRP Disease Activity Score in 28 joints with C-reactive protein (DAS28-CRP); MTX methotrexate

drug-free remission. Age, sex and other factors have been shown to predict better treatment outcomes, but it is unlikely that any of these impacted this analysis. However, as the number of patients is small in this post hoc subgroup analysis, the results reveal only numerical trends, thus requiring further studies for corroboration.

Numerous studies have shown that earlier intervention is associated with better treatment outcomes $[2,3,8]$, leading to the proposal that an optimal therapeutic window is present in early RA. Two large clinical cohorts of patients treated with MTX, using DMARD-free sustained remission as the outcome, suggest that a period exists within the first 6 months in which RA is particularly susceptible to modification with treatment [7]. The findings from our analysis are consistent with such a model in which an early "window of opportunity' exists to achieve the best outcomes. Moreover, the use of abatacept as a first-line bDMARD following an inadequate response to MTX has been shown to be more cost-effective than the use of adalimumab at this stage, in anti-citrullinated protein antibody-positive patients with RA [9].

Our finding that abatacept plus MTX provides the greatest benefit in those with the shortest symptom duration at treatment initiation complements existing data that demonstrate better outcomes with abatacept in early vs established disease ( $\leq 2$ years vs $\geq 10$ years) [8] and further suggest that within early RA there is an optimum treatment window for RA disease modification. The enhanced benefits of abatacept in early RA are likely due to its impact on naïve T cells and upstream mechanism of action which leads to the downregulation of autoantibodies and inflammatory mediators downstream [5]. Research has also demonstrated the ability of abatacept to bind directly to osteoclast precursor cells thereby inhibiting their differentiation, suggesting a protective effect on structural bone damage [10].

RA progresses rapidly in some patients and treatments that are able to achieve disease control quickly are greatly needed to prevent irreversible joint damage and disability. The AVERT study patient population had highly active disease and poor prognostic markers, indicative of a high risk of structural damage and disease progression [5]. We found that, in this patient population, the onset of remission was quickest in those with symptom duration $\leq 3$ months, again demonstrating the benefits of earlier intervention. Indeed, faster response to treatment with a tumor necrosis factor inhibitor [11] and lower disease activity within 3 months of initiating treatment [6] have been shown to be indicative of better long-term outcomes.

The results of the AVERT trial, indicating that abatacept can achieve drug-free remission in patients with very early disease [5], are consistent with the finding that abatacept has a greater impact on naïve $\mathrm{T}$ cells than on memory $\mathrm{T}$ cells [12], providing a scientific rationale for the use of abatacept early in the disease course.

As adverse events are always a concern when using medications, allowing patients in remission to discontinue treatment without relapse would be advantageous. In addition, maintenance of drug-free remission would provide significant financial benefits to payers as well as patients. In this study, we were not able to predict which patients were most likely to achieve remission and stay in remission. This should be a goal of future research.

Limitations of this study should be considered and include that this was a post hoc analysis, and that the trial was not powered to detect the effects of abatacept monotherapy. In addition, there may be a regional difference in how quickly patients access care across the symptom duration groups. A greater number of males in the shorter symptom duration group may also have influenced a better prognosis [13], and a lower baseline HAQ may indicate that patients were less impacted by the disease and thus more inclined to stay off therapy. While a 6-month window is frequently used for similar analyses, here we used a 3-month window to examine the very earliest patients, which revealed more clear effects compared with the longer duration groups ( $>3$ to $\leq 6$ months and $>6$ months). In addition, we used a $>0.3$-unit cut-off point for our minimal clinically important difference of the HAQ-DI (others have used a 0.22 or 0.4 change $[14,15]$ ), as this had been a previously defined threshold for analysis in this trial and supported by the literature [5, 16].

In summary, early treatment of patients with early, active, poor prognosis RA with abatacept plus MTX results in a numerically higher rate of remission and improvements in physical function compared with MTX monotherapy, with the fastest onset and greatest benefit to patients with the shortest symptom duration ( $\leq 3$ months). 


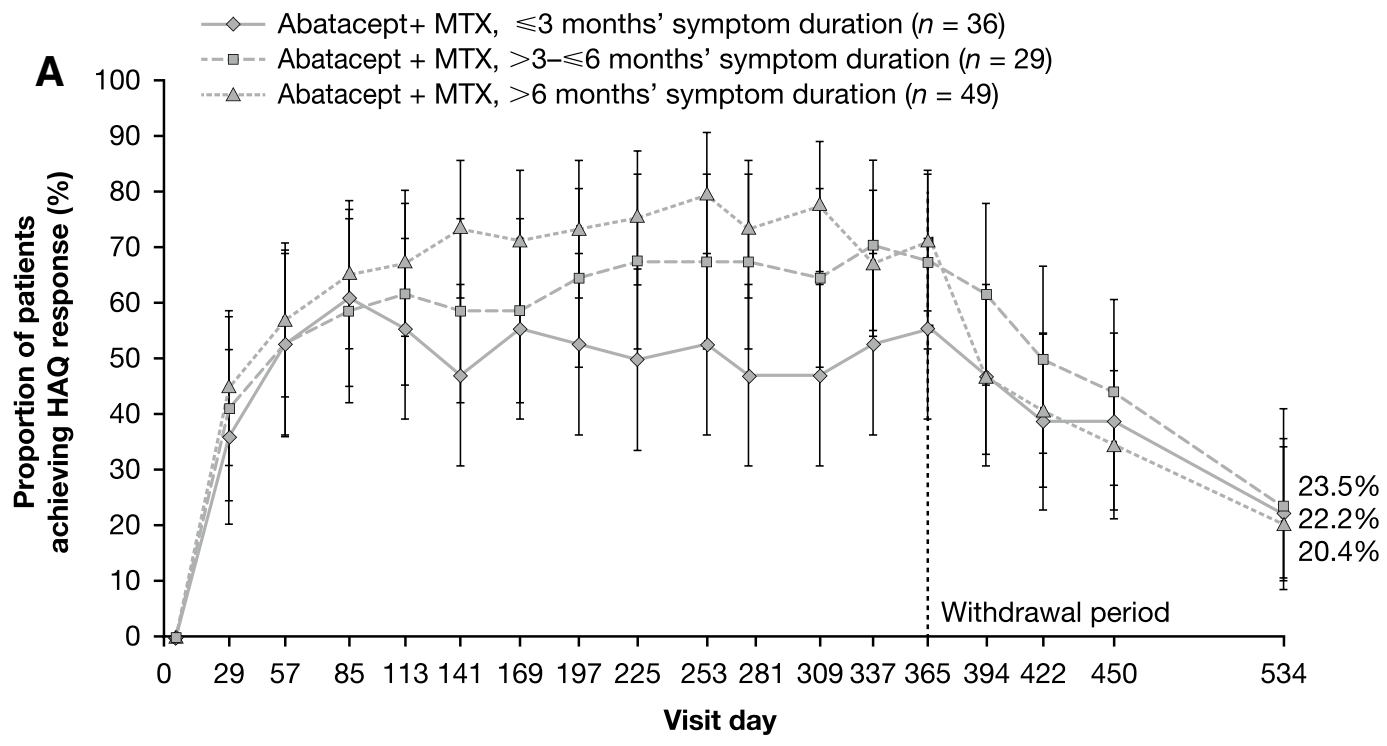

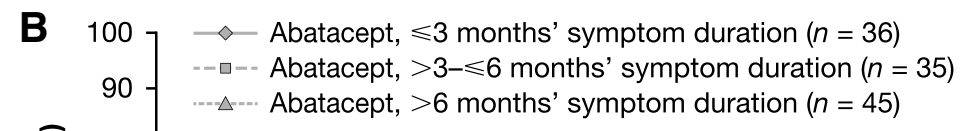
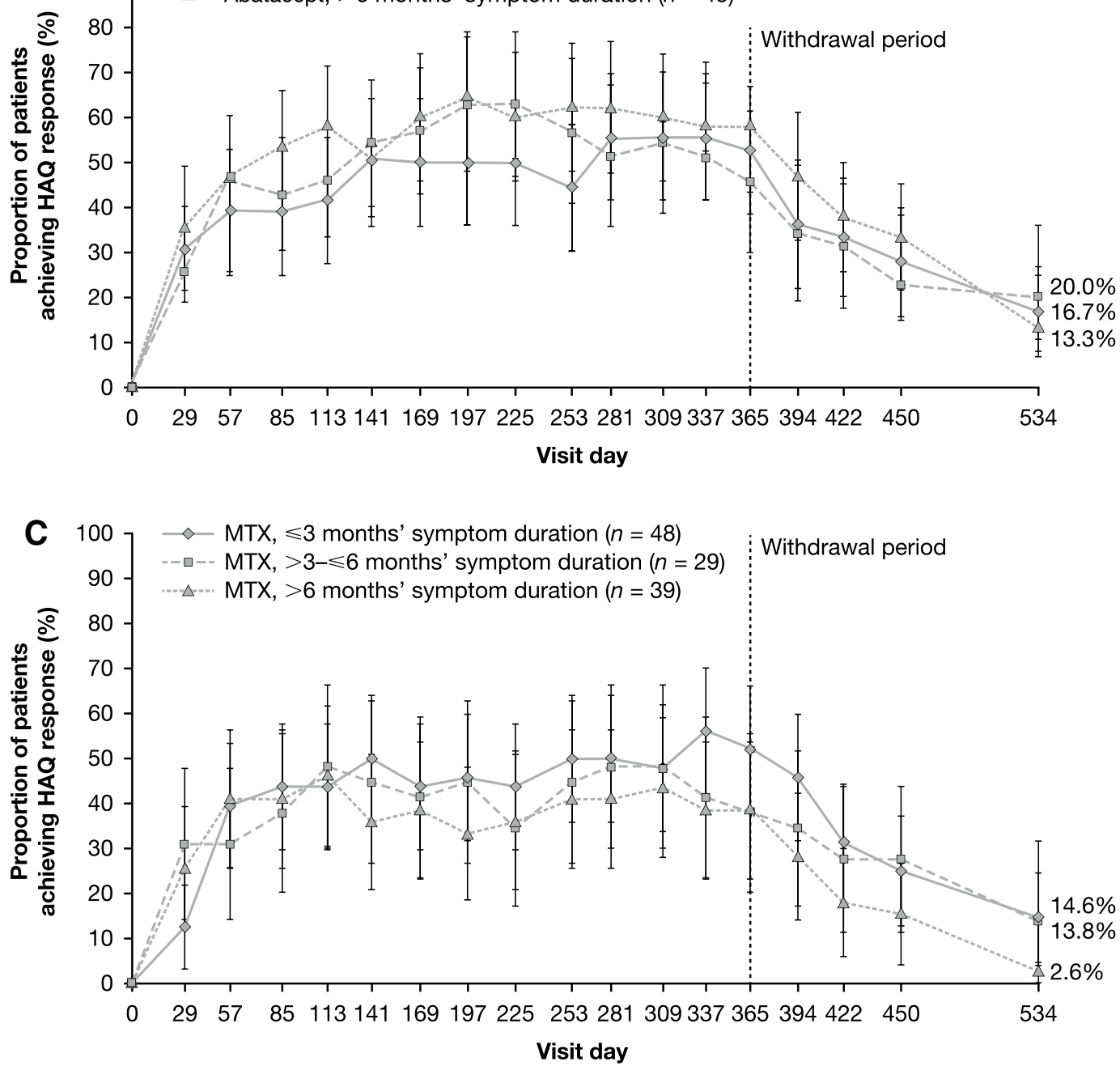
4Fig. 2 Proportion of patients achieving HAQ response over time by baseline symptom duration in each treatment arm: a abatacept + MTX, b abatacept monotherapy, c MTX monotherapy. Error bars represent $95 \%$ CI. HAQ response is defined as a reduction of at least 0.30 units from baseline. $H A Q$ Health Assessment Questionnaire; $M T X$ methotrexate

Acknowledgements Professional medical writing and editorial assistance was provided by Sean Sheffler-Collins, $\mathrm{PhD}$, and Stacey Reeber, $\mathrm{PhD}$, at Caudex and was funded by Bristol-Myers Squibb. The authors would like to thank Dennis Wong, formerly of Bristol-Myers Squibb, for his assistance with this study. Bristol-Myers Squibb, the study sponsor, designed the study in collaboration with the academic authors, conducted the study, collected the data, performed statistical analyses, and were involved with the writing of the study manuscript.

Author contributions In accordance with ICMJE criteria, all authors were involved in writing and drafting the article or revising it critically for important intellectual content. All authors approved the final version to be submitted for publication and agree to be accountable for all aspects of the work. Dr Bykerk had full access to all data in the study and takes responsibility for the integrity of the data and the accuracy of the data analysis. Study conception and design. Bykerk, Burmester, Combe, Furst, Huizinga, Ahmad, Emery. Acquisition of data. Bykerk, Emery. Analysis and interpretation of data. Bykerk, Burmester, Combe, Furst, Huizinga, Emery.

Funding This work was supported by Bristol-Myers Squibb.

\section{Compliance with ethical standards}

Ethical standards The study was conducted in accordance with the Declaration of Helsinki and was approved by local institutional review boards or Independent Ethics Committees at each site (ClinicalTrials.gov; NCT01142726). The laws and regulatory requirements of all countries participating in this study were followed.

Informed consent All patients provided written informed consent.

Open Access This article is distributed under the terms of the Creative Commons Attribution 4.0 International License (http://creativeco mmons.org/licenses/by/4.0/), which permits unrestricted use, distribution, and reproduction in any medium, provided you give appropriate credit to the original author(s) and the source, provide a link to the Creative Commons license, and indicate if changes were made.

\section{References}

1. Combe B (2009) Progression in early rheumatoid arthritis. Best Pract Res Clin Rheumatol 23:59-69

2. Cush JJ (2007) Early rheumatoid arthritis—is there a window of opportunity? J Rheumatol Suppl 80:1-7

3. Anderson JJ, Wells G, Verhoeven AC, Felson DT (2000) Factors predicting response to treatment in rheumatoid arthritis: the importance of disease duration. Arthritis Rheum 43:22-29

4. Emery P, Durez P, Dougados M, Legerton CW, Becker JC, Vratsanos G, Genant HK, Peterfy C, Mitra P, Overfield S, Qi K, Westhovens R (2010) Impact of T-cell costimulation modulation in patients with undifferentiated inflammatory arthritis or very early rheumatoid arthritis: a clinical and imaging study of abatacept (the ADJUST trial). Ann Rheum Dis 69:510-516

5. Emery P, Burmester GR, Bykerk VP, Combe BG, Furst DE, Barre E, Karyekar CS, Wong DA, Huizinga TW (2015) Evaluating drugfree remission with abatacept in early rheumatoid arthritis: results from the phase $3 \mathrm{~b}$, multicentre, randomised, active-controlled AVERT study of 24 months, with a 12-month, double-blind treatment period. Ann Rheum Dis 74:19-26

6. Aletaha D, Funovits J, Keystone EC, Smolen JS (2007) Disease activity early in the course of treatment predicts response to therapy after one year in rheumatoid arthritis patients. Arthritis Rheum 56:3226-3235

7. van Nies JA, Tsonaka R, Gaujoux-Viala C, Fautrel B, AH vdH-vM (2015) Evaluating relationships between symptom duration and persistence of rheumatoid arthritis: does a window of opportunity exist? Results on the Leiden early arthritis clinic and ESPOIR cohorts. Ann Rheum Dis 74:806-812

8. Yazici Y, Moniz RD, Klem C, Rosenblatt L, Wu G, Kremer JM (2011) Greater remission rates in patients with early versus longstanding disease in biologic-naive rheumatoid arthritis patients treated with abatacept: a post hoc analysis of randomized clinical trial data. Clin Exp Rheumatol 29:494-499

9. Neubauer AS, Minartz C, Herrmann KH, Baerwald C (2018) Cost-effectiveness of early treatment of ACPA-positive rheumatoid arthritis patients with abatacept. Clin Exp Rheumatol 36:448-454

10. Axmann R, Herman S, Zaiss M, Franz S, Polzer K, Zwerina J, Herrmann M, Smolen J, Schett G (2008) CTLA-4 directly inhibits osteoclast formation. Ann Rheum Dis 67:1603-1609

11. Keystone EC, Curtis JR, Fleischmann RM, Furst DE, Khanna D, Smolen JS, Mease PJ, Schiff MH, Coteur G, Davies O, Combe B (2011) Rapid improvement in the signs and symptoms of rheumatoid arthritis following certolizumab pegol treatment predicts better longterm outcomes: post-hoc analysis of a randomized controlled trial. J Rheumatol 38:990-996

12. Cutolo M, Nadler S (2013) Advances in CTLA-4-Ig-mediated modulation of inflammatory cell and immune response activation in rheumatoid arthritis. Autoimmun Rev 12:758-767

13. Kuriya B, Xiong J, Boire G, Haraoui B, Hitchon C, Pope J, Thorne JC, Tin D, Keystone EC, Bykerk V (2014) Earlier time to remission predicts sustained clinical remission in early rheumatoid arthritis-results from the Canadian Early Arthritis Cohort (CATCH). J Rheumatol 41:2161-2166

14. Strand V, Balbir-Gurman A, Pavelka K, Emery P, Li N, Yin M, Lehane PB, Agarwal S (2006) Sustained benefit in rheumatoid arthritis following one course of rituximab: improvements in physical function over 2 years. Rheumatology 45:1505-1513

15. Maska L, Anderson J, Michaud K (2011) Measures of functional status and quality of life in rheumatoid arthritis: Health Assessment Questionnaire Disability Index (HAQ), Modified Health Assessment Questionnaire (MHAQ), Multidimensional Health Assessment Questionnaire (MDHAQ), Health Assessment Questionnaire II (HAQ-II), Improved Health Assessment Questionnaire (Improved HAQ), and Rheumatoid Arthritis Quality of Life (RAQoL). Arthritis Care Res (Hoboken) 63(Suppl 11):S4-S13

16. Coombs JH, Bloom BJ, Breedveld FC, Fletcher MP, Gruben D, Kremer JM, Burgos-Vargas R, Wilkinson B, Zerbini CA, Zwillich SH (2010) Improved pain, physical functioning and health status in patients with rheumatoid arthritis treated with CP-690,550, an orally active Janus kinase (JAK) inhibitor: results from a randomised, double-blind, placebo-controlled trial. Ann Rheum Dis 69:413-416 